\title{
Geriatric Pharmacology: A Complex World of Choices
}

\author{
Frisardi $\mathbf{V}^{1,2^{*}}$ and Zaccaria $\mathbf{D}^{1,3}$ \\ ${ }^{1}$ Golgi-Cenci Foundation, Center for Brain and Aging Research, Abbiategrasso (MI), Italy \\ ${ }^{2}$ Golgi Redaelli, Geriatric-Functional and Cognitive Rehabilitation- Institute, Abbiategrasso (MI), Italy \\ ${ }^{3}$ Department of Sociology and Social Research, University of Milano, Bicocca, Italy \\ *Correspondence Author: Vincenza Frisardi, Piazza C. Golgi 11, 20081, Abbiategrasso, Milan, Italy, Tel: +39 0294852539 ; \\ E-mail: vfrisardi@yahoo.com
}

Rec date: Sep 30, 2014; Acc date: Oct 5, 2014; Pub date: Oct 15, 2014

Copyright: (c) 2014 Frisardi V. This is an open-access article distributed under the terms of the Creative Commons Attribution License, which permits unrestricted use, distribution, and reproduction in any medium, provided the original author and source are credited.

\section{Editorial}

It is expected that by 2050 Americans 65 or older will be almost 89 million or more and then more than double the number of older adults in the United States in 2010 [1]. While elders make up less than $13 \%$ of the U.S. population, they have become the leading drug consumers [2]. With the increasing aging population, this number will increase dramatically over the next 30 years, necessitating a larger focus on geriatric medication issues and an increase in geriatric-competent clinicians to ensure optimal care. Identifying, preventing, and resolving medication-related problems will become increasingly important to the stability of our healthcare system and welfare.

Several issues emerge about pharmacology in older people, ranging from "improper drug use without indication" to subtherapeutic dosage or overdosage, as well as adverse drug reactions, drug-drug interactions, compliance to treatment and polypharmacy. Sometimes these problems come from an incomplete knowledge about drugs metabolism. Developing an effective pharmacotherapeutic plan for an elderly patient requires a clear understanding of the principles of pharmacokinetics (absorption, distribution, metabolism, and elimination) and how the pharmacokinetics of a drug may be altered in the geriatric population. However, apart pharmacokinetic and pharmacodynamics changes age-related, there is a fascinating alternative field of research (pharmacogenomics) that explored the underlying mechanisms of drugs metabolism. In fact, it has been demonstrated that the absorption and utilization of several medication depending on different cytochrome p450 (CYP450) polymorphisms. The CYP450 system is the site where many drug- drug interactions occur, because various drugs can act as inducers or inhibitors of other drugs undergoing metabolism and altering pharmacokinetics of several compounds [3]. Furthermore, it has been demonstrated that genetic polymorphisms contribute to effectiveness variation of medications by specific receptors' constitutional modifications. For instance, antidepressant therapy appears to be influenced by the polymorphism of the serotonin transporter [4].

Nowadays, many medications have recommended geriatric maximum doses, frequency, and duration of use while others are not recommended for use in the elderly. The Beers Criteria revolutionized geriatric medication use, because have indicated a list of medications that should potentially be avoided in elders or avoided in the presence of certain comorbidities. The list was initiated in 1991 and an update was completed by the American Geriatrics Society [5]. The criteria are intended to provide physicians and healthcare providers a comprehensive list of medications that may be harmful to older adult patients. The updated criteria are divided in three detailed lists. The first list is classified as problematic drugs in elderly adults. The second as "inappropriate for older people who have certain diseases or risk factors. The third classification lists as "alarming drugs", that is, that should be used with caution in elderly patients. Most of the medications on the lists are well known, and medical evidence supports their potential to be harmful in elderly. The Beers criteria does not penalize the use of drugs on the list for all elderly patients; instead, they emphasize the drugs that may pose the most harm so that they are used judiciously and with caution. Older people are more exposed to multiple comorbidities and their significant functional implications. The response of physicians to these clinical conditions is often to prescribe several medications, although the pharmacological response is highly variable and health outcomes are not always achieved. In fact, polypharmacy, defined as the use of five or more medications, occurs in almost $40-50 \%$ of older people and has been linked to poor health outcomes [2]. Many times polypharmacy is inevitable but sometimes it occurs simply because healthcare providers fail to communicate proper patient recommendations to the patient's primary care provider. If each patient had a primary healthcare advocate who coordinated the patient's overall care, or his general practitioner who periodically updated the treatment plan according to the state of health of the patient, then the risk of polypharmacy could be reduced.

Similar to but much more comprehensive than the Beers criteria is the Screening Tool of Older Persons' Prescriptions. Rather than a simple checklist of medications, it attempts to assess the use of a drug with a specific patient who has specific comorbidities [5]. Further efforts need to be made in this field of research with the aim to include psychosocial and economic aspects that dictate treatment decisions and patient compliance. In fact, although most patients are motivated to take their medications as prescribed, some may fail to comply due to lack of understanding, confusion, forgetfulness or simple because they refuse to take so many pills. Obviously, the line between use and abuse of prescribed drugs is very thin and the topic is very attractive for health and welfare policies. Ensuring optimal medication use requires careful coordination of care and communication among prescribers, other healthcare providers, and patients and their caregivers. Whichever tool is used, the primary purpose is to prescribe medications to all elderly patients with care and consideration, which ensures that each older adult patient is provided with the best care possible. Achieving this goal will result in healthy patients, less polypharmacy, fewer adverse drug effects, and lower healthcare costs. At present, the type of pharmacotherapy that is available to counteract diseases is extensive. The number of available drugs will continue to increase. In elderly, these drugs are effective only if used reasonably with a multidimensional approach regarding the appropriateness, efficacy and effectiveness of therapy. An attentive consideration about 
Citation: Frisardi V, Zaccaria D (2014) Geriatric Pharmacology: A Complex World of Choices. J Gerontol Geriatr Res 3: 1000E133. doi: 10.4172/2167-7182.1000E133

Page 2 of 2

every pharmacotherapy plan will help ensure that all patients receive the best care possible.

\section{References}

1. US Census Bureau. National population projections.

2. Jörgensen $T$, Johansson $S$, Kennerfalk A, Wallander MA, Svärdsudd K (2001) Prescription drug use, diagnoses, and healthcare utilization among the elderly. Ann Pharmacother 35: 1004-1009.

3. Savino M, Seripa D, Gallo AP, Garrubba M, D'Onofrio G, et al. (2011) Effectiveness of a high-throughput genetic analysis in the identification of responders/non-responders to CYP2D6-metabolized drugs. Clin Lab 57: 887-893.

4. Matsumoto Y, Fabbri C, Pellegrini S, Porcelli S, Politi P, et al. (2014) Serotonin transporter gene: a new polymorphism may affect response to antidepressant treatments in major depressive disorder. Mol Diagn Ther 18: 567-577.

5. Blanco-Reina E, Ariza-Zafra G, Ocaña-Riola R, León-Ortiz M (2014). 2012 American Geriatrics Society Beers criteria: enhanced applicability for detecting potentially inappropriate medications in European older adults? A comparison with the Screening Tool of Older Person's Potentially Inappropriate Prescriptions. J Am Geriatr Soc 62:1217-1223. 